\title{
Numerical Continuation of Fold Bifurcations of Limit Cycles in MATCONT
}

\author{
Annick Dhooge $^{1}$, Willy Govaerts ${ }^{1}$, and Yuri A. Kuznetsov ${ }^{2}$ \\ 1 Department of Applied Mathematics and Computer Science, Ghent University, \\ Krijgslaan 281-S9,B-9000 Gent, Belgium \\ \{Annick.Dhooge,Willy.Govaerts\}@rug.ac.be \\ 2 Mathematisch Instituut, Universiteit Utrecht, \\ Boedapestlaan 6, 3584 CD Utrecht, The Netherlands \\ kuznetsov@math.uu.nl
}

\begin{abstract}
MATCONT is a Matlab continuation package with a GUI for the interactive numerical study of a range of parameterized nonlinear problems. In the case of ODEs it allows to compute curves of equilibria, limit points, Hopf points, limit cycles, period doubling bifurcation points of limit cycles and fold bifurcation points of limit cycles. It can use the symbolic toolbox of Matlab to compute derivatives of order up to five of the object function. We discuss some details on the implementation of the limit cycles and the fold bifurcation of limit cycles in MATCONT. The inherent sparsity of the discretized systems for the computation of limit cycles and their bifurcation points is exploited by using the standard Matlab sparse matrix methods.
\end{abstract}

\section{Introduction}

Numerical continuation is a well - understood subject, see e.g. [1], 2], 8], 10]. The idea is as follows. Consider a smooth function $F: \mathbb{R}^{n+1} \rightarrow \mathbb{R}^{n}$. We want to compute a solution curve of the equation $F(x)=0$. Numerical continuation is a technique to compute a sequence of points which approximate the desired branch.

In particular, we consider a dynamical system of the form

$$
\frac{d x}{d t}=f(x, \alpha)
$$

with $x \in \mathbb{R}^{n}, f(x, \alpha) \in \mathbb{R}^{n}$, and $\alpha$ a vector of parameters where equilibria, limit points, limit cycles etcetera can be computed.

The existing software packages such as AUTO [6], CONTENT [11] require the user to rewrite his/her models in a specific format; this complicates the export of results, graphical representation etcetera.

The aim of MATCONT is to provide a continuation toolbox which is compatible with the standard Matlab ODE representation of differential equations. This toolbox is developed with the following targets in mind: 
- detection of singularities via test functions

- singularity-specific location code

- processing of regular and singular points

- support of adaptive meshes

- support of symbolic derivatives

- support for sparse matrices

Earlier versions of the toolbox are described in 15 13. The current version of the package is freely available for download at:

http://allserv.rug.ac.be/ ajdhooge/research.html

It requires Matlab 6.* to be installed on your computer. In the present paper we concentrate on the implementation in MATCONT of the continuation of the Fold bifurcation of limit cycles, using a minimal extended system, i.e. we only append a scalar equation to the definition of limit cycles [7]. The only existing software to perform this continuation is AUTO97-00 [6] which uses a maximal extended system, i.e. the number of state variables is approximately doubled. In the somewhat similar situation of the continuation of the period doubling bifurcations it was shown in [7] that at least in the Matlab implementation minimal extended systems are much more efficient. Details on the general structure of MATCONT are given in [5]. We show its use in a relatively simple situation where we continue an equilibrium, a limit point and a Hopf point of a differential equation defined in a standard Matlab ODE file. This file is built using the GUI of MATCONT (Fig. 1, left). We note that the standard Matlab odeget and odeset only support Jacobian matrices w.r.t. phase variables coded in the ode-file. However, we also need the derivatives with respect to the parameters. It is also useful to have higher-order symbolic derivatives available. To overcome this problem, the package contains new versions of odeget and odeset which support Jacobians with respect to parameters (Jacobianp) and higher-order derivatives. The new routines are compatible with the ones provided by Matlab. The GUI of matcont makes it possible to choose the option to compute the derivatives symbolically if the symbolic toolbox is installed (Fig. 1, left).

The following chemical model of a catalytic reaction of $\mathrm{CO}$-oxidation was studied in 3] and 9].

$$
\left\{\begin{array}{l}
\dot{x}=2 q_{1} z^{2}-2 q_{5} x^{2}-q_{3} x y \\
\dot{y}=q_{2} z-q_{6} y-q_{3} x y \\
\dot{z}=-q_{4} z-k q_{4} s
\end{array}\right.
$$

where $z=1-x-y-s$. In this low-dimensional problem a curve of equilibrium solutions was traced with $q_{2}$ as free parameter, starting from the initial values $q_{1}=2.5, q_{2}=1.040992, q_{3}=10, q_{4}=0.0675, q_{5}=1, q_{6}=0.1, k=0.4, x=$ $0.07792759, y=0.2330654$ and $z=0.4921479$. This branch is represented in Fig. 1 (left) as the curve that starts in the bottom right corner. On this equilibrium branch two limit points (LP) and two Hopf points $(\mathrm{H})$ were found.

The first Hopf point is used to start a Hopf curve continuation with free parameters $q_{2}$ and $k$. We find a closed curve that connects the two Hopf points. 


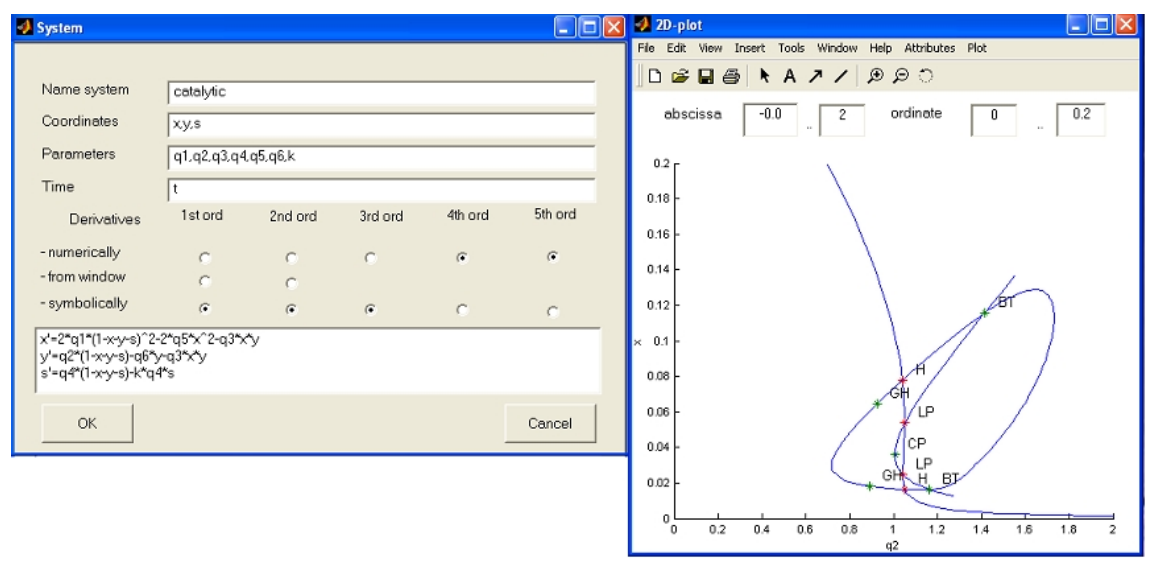

Fig. 1. The system window and a bifurcation diagram.

The symbol BT on Fig. 1(right) denotes a Bogdanov-Takens point. The two GH points in Fig. 1(right) are generalized Hopf points, i.e., points where the first Lyapunov coefficient changes sign. The first limit point is used to start a forward fold continuation with both $q_{2}$ and $k$ free. Both limit points on the equilibrium curve are connected by this fold curve. This fold curve also contains a Cusp point (CP) and intersects the Hopf curve in the BT points.

\section{$2 \quad$ Limit Cycles and Fold Bifurcations of Limit Cycles}

\subsection{Limit Cycles}

A limit cycle is an isolated periodic solution of (1) with period $T$, i.e. $x(0)=x(T)$.

Since $T$ is not known in advance we use an equivalent system defined on the fixed interval $[0,1]$ by rescaling time. Then the system reads

$$
\left\{\begin{array}{l}
\frac{d x}{d t}-T f(x, \alpha)=0 \\
x(0)=x(1)
\end{array}\right.
$$

A phase shifted function $\phi(t)=x(t+s)$ is also a solution of (2) for any value of $s$. To obtain a unique solution an extra constraint is needed. The following integral constraint is often used [6, $[1]$

$$
\int_{0}^{1}\left\langle x(t), \dot{x}_{\text {old }}(t)\right\rangle d t=0
$$

where $\dot{x}_{\text {old }}(t)$ is the tangent vector of a previously calculated limit cycle and is therefore known, $\langle x, v\rangle$ is just a different notation for $x^{T} v$. This condition tries to select the solution which has the smallest phase difference with respect to the previous solution $x_{\text {old }}$.

The complete boundary value problem (BVP) defining a limit cycle consists of (2) and (3). 


\subsection{Fold Bifurcation of Limit Cycles}

A Fold bifurcation of limit cycles (Limit Point of Cycles, LPC) generically corresponds to a turning point of a curve of limit cycles (cf. the example in \$5). It can be characterized by adding an extra constraint $G=0$ to (2), (3) where $G$ is the Fold test function. The complete BVP defining a LPC point using the minimal extended system is

$$
\begin{cases}\frac{d x}{d t}-T f(x, \alpha) & =0 \\ x(0)-x(1) & =0 \\ \int_{0}^{1}\left\langle x(t), \dot{x}_{\text {old }}(t)\right\rangle d t & =0 \\ G[x, T, \alpha] & =0\end{cases}
$$

where $G$ is defined by requiring

$$
N^{1}\left(\begin{array}{c}
v \\
S \\
G
\end{array}\right)=\left(\begin{array}{l}
0 \\
0 \\
0 \\
1
\end{array}\right) .
$$

Here $v$ is a function, $S$ and $G$ are scalars and

$$
N^{1}=\left[\begin{array}{ccc}
D-T f_{x}(x(t), \alpha) & -f(x(t), \alpha) & w_{01} \\
\delta_{1}-\delta_{0} & 0 & w_{02} \\
\operatorname{Int}_{f(x(\cdot), \alpha)} & 0 & w_{03} \\
\operatorname{Int}_{v_{01}} & v_{02} & 0
\end{array}\right]
$$

where the bordering functions $v_{01}, w_{01}$, vector $w_{02}$ and scalars $v_{02}$ and $w_{03}$ are chosen so that $N^{1}$ is nonsingular [7].

\section{Continuation of Limit Cycles}

For the numerical continuation of a limit cycle with respect to a parameter we need to discretize the system consisting of (2) and (3). And to use a Newton-like method the Jacobian matrix of the discretized system is also needed.

\subsection{Discrete Representation of the Solution Function}

The method used to discretize the BVP is called orthogonal collocation [4]. First the interval [ $\left[\begin{array}{ll}0 & 1\end{array}\right]$ is subdivided in $N$ smaller intervals.

$$
0=\tau_{0}<\tau_{1}<\cdots<\tau_{N}=1 .
$$

On each of these intervals the solution $x(\tau)$ is approximated by an order $m$ vector valued polynomial $x^{(i)}(\tau)$. This is done by defining $m+1$ equidistant points on each interval

$$
\tau_{i, j}=\tau_{i}+\frac{j}{m}\left(\tau_{i+1}-\tau_{i}\right)(j=0,1, \ldots, m)
$$

and defining the polynomials $x^{(i)}(\tau)$ as 


$$
x^{(i)}(\tau)=\sum_{j=0}^{m} x^{i, j} l_{i, j}(\tau) .
$$

Here $x^{i, j}$ is the discretization of $x(\tau)$ at $\tau=\tau_{i, j}$ (we note that $x^{N-1, m}=x^{N, 0}$ ) and the $l_{i, j}(\tau)$ 's are the Lagrange basis polynomials

$$
l_{i, j}(\tau)=\prod_{k=0, k \neq j}^{m} \frac{\tau-\tau_{i, k}}{\tau_{i, j}-\tau_{i, k}}
$$

On each interval $\left[\tau_{i}, \tau_{i+1}\right]$ we require that the polynomials $x^{(i)}(\tau)$ satisfy the BVP exactly in $m$ collocation points $\zeta_{i, j}(j=1, \ldots, m)$ (which are not the same as the $\tau_{i, j}$ ). It can be proved that the best choice for the collocation points are the Gauss points [4. These are the roots of the Legendre polynomial relative to the interval $\left[\tau_{i}, \tau_{i+1}\right]$. With this choice of collocation points the error in the approximation is extremely small

$$
\left\|x\left(\tau_{i, j}\right)-x^{i, j}\right\|=\mathcal{O}\left(h^{m}\right)
$$

and for the mesh points $\tau_{j}$ it's even better

$$
\left\|x\left(\tau_{i}\right)-x^{i, 0}\right\|=\mathcal{O}\left(h^{2 m}\right) .
$$

\subsection{Numerical Integration}

\begin{tabular}{|c|c|c|c|c|c|c|}
\hline$\tau_{0}$ & & $\tau_{1}$ & & $\tau_{2}$ & & $\tau_{3}$ \\
\hline 0 & 0 & $\bullet$ & 0 & $\bullet$ & ○ & 0 \\
\hline \multirow[t]{2}{*}{$\tau_{0,0}$} & $\tau_{0,1}$ & $\tau_{0,2}$ & & $\tau_{2,0}$ & $\tau_{2,1}$ & $\tau_{2,2}$ \\
\hline & & $\tau_{1,0}$ & $\tau_{1,1}$ & $\tau_{1,2}$ & & $\tau_{3,0}$ \\
\hline$\sigma_{0,0}$ & $\sigma_{0,1}$ & $\sigma_{1,0}$ & $\begin{array}{l}\sigma_{2} \omega_{2} \\
\sigma_{1,1}\end{array}$ & $\sigma_{2,0}$ & $\sigma_{21}$ & $\sigma_{3,0}$ \\
\hline
\end{tabular}

In (4) and (5) we need to compute integrals over [0 1] using the discretization discussed in 3.1 For $N=3$ test intervals and $m=2$ collocation points the following data are associated with the discretized interval [ $\left[\begin{array}{ll}0 & 1\end{array}\right]$

The total number of mesh points (tps) is $N \times m+1$, the total number of points (ncoords) is tps $\times \operatorname{dim}(x)$. Each mesh point $\tau_{i, j}$ in a test interval $\left[\tau_{i}, \tau_{i+1}\right]$ has a particular weight $w_{j+1}$, the Gauss-Lagrange quadrature coefficient. Some mesh points (the black bullets) belong to two test intervals. We set $t_{i}=\tau_{i}-\tau_{i-1}(i=$ $1, \ldots, N)$. The integration weight $\sigma_{i, j}$ of $\tau_{i, j}$ is given by $w_{j+1} t_{i+1}$ for $0 \leq i \leq N-1$ and $0<j<m$. For $i=0, \ldots, N-2$ the integration weight of $\tau_{i, m}=\tau_{i+1,0}$ is given by $\sigma_{i, m}=w_{m+1} t_{i+1}+w_{1} t_{i+2}$ and the integration weights of $\tau_{0}$ and $\tau_{N}$ are given by $w_{1} t_{1}$ and $w_{m+1} t_{N}$, respectively. The integral $\int_{0}^{1} f(t) d t$ is approximated by $\sum_{i=0}^{N-1} \sum_{j=0}^{m-1} f\left(\tau_{i, j}\right) \sigma_{i, j}+f(1) \sigma_{N, 0}$. 


\subsection{Discretization of the BVP}

Using the discretization described in $\$ 3.1$ we obtain the discretized BVP

$$
\left\{\begin{array}{r}
\left(\sum_{j=0}^{m} x^{i, j} l_{i, j}^{\prime}\left(\zeta_{i, k}\right)\right)-T f\left(\sum_{j=0}^{m} x^{i, j} l_{i, j}\left(\zeta_{i, k}\right), \alpha\right)=0 \\
x^{0,0}-x^{N-1, m}=0 \\
\sum_{i=0}^{N-1} \sum_{j=0}^{m-1} \sigma_{i, j}\left\langle x^{i, j}, \dot{x}_{o l d}^{i, j}\right\rangle+\sigma_{N, 0}\left\langle x^{N, 0}, \dot{x}_{\text {old }}^{N, 0}\right\rangle=0
\end{array}\right.
$$

The first equation in fact consists of $\mathrm{Nm}$ equations, one for each combination of $i=0,1,2, \ldots, N-1$ and $k=1,2, \ldots, m$.

\subsection{The Jacobian of the Discretized Equations}

The Jacobian of the discretized system is sparse. In the Newton iterations during the continuation process a system consisting of this Jacobian matrix and an extra row (the tangent vector) is solved. For $N=3$ test intervals, $m=2$ collocation points and $\operatorname{dim}(x)=2$ this matrix has the following sparsity structure $(\bullet$ 's are non-zero's).

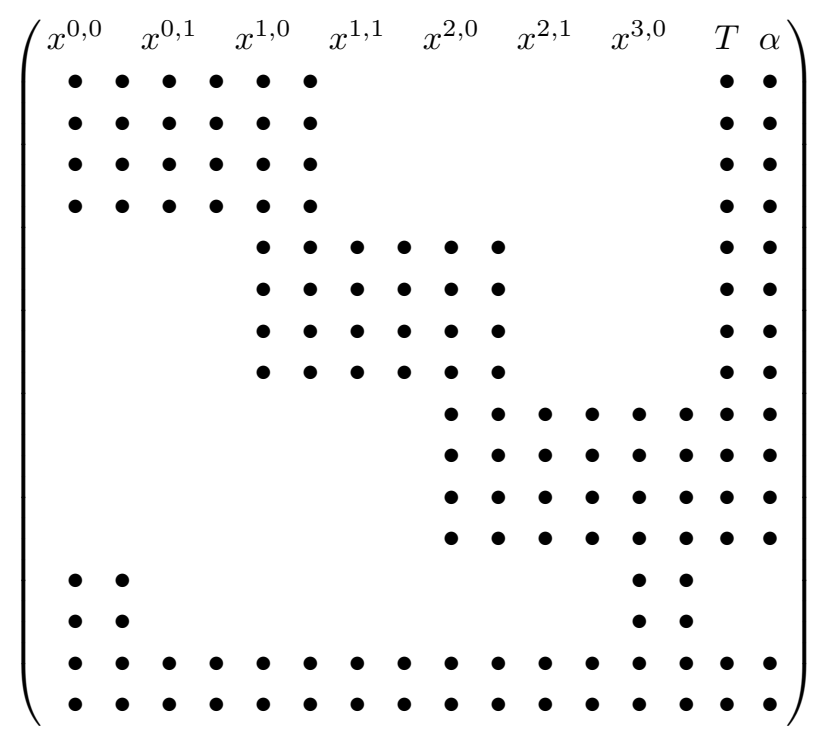

The columns of (7) label the unknowns of the discretized problem. The first $\operatorname{dim}(x)$ rows correspond to the first collocation point etc. In (21) and (3) there are 3 unknowns: $x$, the period $T$ and a parameter $\alpha$. So the part of the Jacobian corresponding with the first equation of (2) has the following form:

$$
\left[D-T f_{x}(x, \alpha) \quad-f(x, \alpha) \quad-T f_{\alpha}(x, \alpha)\right] .
$$

In (7) $D-T f_{x}(x, \alpha)$ corresponds to $N=3$ blocks with dimension $4 \times 6$ (= $(\operatorname{dim}(x) * m) \times(\operatorname{dim}(x) \times(m+1)))$. The part in (7) that defines the boundary conditions for limit cycles has the form:

$$
\left[I_{\operatorname{dim}(x)} \quad 0_{\operatorname{dim}(x) \times(N m-1) \operatorname{dim}(x)} \quad-I_{\operatorname{dim}(x)} \quad 0_{\operatorname{dim}(x)}\right] .
$$


These are in (7) the $\operatorname{dim}(x)=2$ rows following the $4 \times 6$ blocks. These rows contain two nonzero parts corresponding with $x^{0,0}$ and $x^{N, 0}( \pm 2 \times 2$ identity matrix). The last but one row in (7) is the derivative of the discretization of (3). The last row is added by the continuation code.

\section{Continuation of LPC Cycles}

\subsection{Discretization of the LPC Equations}

Numerically we solve a discretized version of (5), say

$$
M_{d}^{1}\left(\begin{array}{c}
v_{d} \\
S_{d} \\
G_{d}
\end{array}\right)=\left(\begin{array}{l}
0 \\
0 \\
0 \\
1
\end{array}\right)
$$

where $M_{d}^{1}$ is the discretization of $N^{1}$ using orthogonal collocation. Here and elsewhere the subscript $d$ denotes discretization. The structure is similar to that of (7); however the two last rows and colums have a different meaning. The last but one row corresponds with $\operatorname{Int}_{[f(x(\cdot), \alpha)]_{d}}$ and the last but one column corresponds with $[-f(x(t), \alpha)]_{d}$.

\subsection{The Jacobian of the Discretized LPC Equations}

To continue the discretized equations of (4) the Jacobian matrix of the system is needed which means that the derivatives of $G_{d}$ with respect to the unknowns of the system, i.e., with respect to $x^{i, j}, T, \alpha$, have to be calculated.

The derivative with respect to $z$ (being a component of $x^{i, j}, T$ or $\alpha$ ) is

$M_{d}^{1}\left(\begin{array}{c}v_{d z} \\ S_{d z} \\ G_{d z}\end{array}\right)+\left[\begin{array}{ccc}\left(\left[-T f_{x}(x(t), \alpha)\right]_{d}\right)_{z} & \left([-f(x(t), \alpha)]_{d}\right)_{z} & 0 \\ 0 & 0 & 0 \\ \left(\operatorname{Int} t_{\left.[f(x(\cdot), \alpha)]_{d}\right)_{z}}\right. & 0 & 0 \\ 0 & 0 & 0\end{array}\right]\left(\begin{array}{c}v_{d} \\ S_{d} \\ G_{d}\end{array}\right)=\left(\begin{array}{c}0 \\ 0 \\ 0 \\ 0\end{array}\right)$.

Simplifying gives

$$
M_{d}^{1}\left(\begin{array}{c}
v_{d z} \\
S_{d z} \\
G_{d z}
\end{array}\right)=\left(\begin{array}{c}
{\left[T f_{x}(x(t), \alpha]_{d z} v_{d}+[f(x(t), \alpha)]_{d z} S_{d}\right.} \\
0 \\
-\operatorname{Int}_{[f(x(\cdot), \alpha)]_{d z}} v_{d} \\
0
\end{array}\right)
$$

Instead of solving this for every $z$ we solve the transposed equations

$$
\left(w_{1}^{*}, w_{2}^{*}, w_{3}, w_{4}\right) M_{d}^{1}=(0,0,1)
$$

where $w_{1}$ is a $\operatorname{dim}(x) * N * m$ vector, $w_{2}$ a $\operatorname{dim}(x)$ vector and $w_{3}$ and $w_{4}$ are scalars. Combining (8) and (9) we find

$$
G_{d z}=w_{1}^{*}\left(\left[T f_{x}(x(t), \alpha]_{d z} v_{d}+[f(x(t), \alpha)]_{d z} S_{d}\right)-w_{3} \operatorname{Int}_{[f(x(\cdot), \alpha)]_{d z}} v_{d} .\right.
$$

So in each iteration step we solve two systems with the structure of (7) or its transpose. We note that the Jacobian is very sparse if $N$ is large; we exploit this by using the Matlab sparse matrix routines. 


\subsection{Multipliers}

Multipliers can be computed in MATCONT as in AUto 6] and CONTENT 11 by making a special decomposition(condensation of parameters) in (7). In LPC points two multipliers must be equal to 1 . This can be used to check the correctness of the continuation.

\section{An Example}

We consider the following system

$$
\left\{\begin{aligned}
\dot{v} & =y-0.5(v+0.5)-2 w(v+0.7)-m_{\infty}(v-1) \\
\dot{w} & =1.15\left(w_{\infty}-w\right) \tau
\end{aligned}\right.
$$

where $m_{\infty}=(1+\tanh ((v+0.01) / 0.15)) / 2, w_{\infty}=(1+\tanh ((v-z) / 0.145)) / 2$ and $\tau=\cosh ((v-0.1) / 0.29)$. Here $v$ and $w$ are the state variables and $y$ and $z$

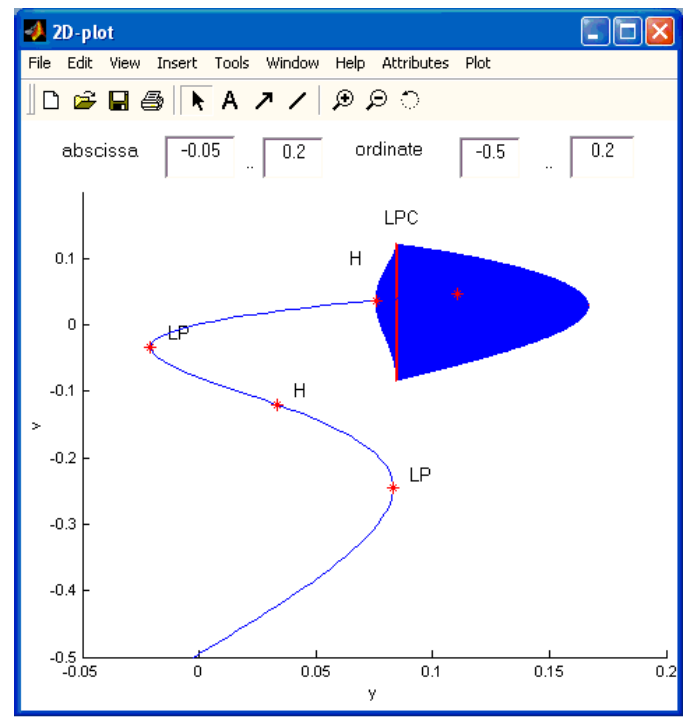

Fig. 2. Plot of equilibrium curve, limit cycles started from $\mathrm{H}$ and the LPC curve started from LPC.

are the parameters. This is a modification of the fast subsystem of the MorrisLecar equations studied in [16],[17]; the Morris-Lecar equations were introduced in [14] as a model for the electrical activity in the barnacle giant muscle fiber. In our model y corresponds to the slow variable in the fast Morris-Lecar equations; $\mathrm{z}$ is the potential that corresponds to $w_{\infty}=\frac{1}{2}$. By time integration (accessible via the MATCONT GUI) we find a stable equilibrium (EP) for $y=0.110472$ and 


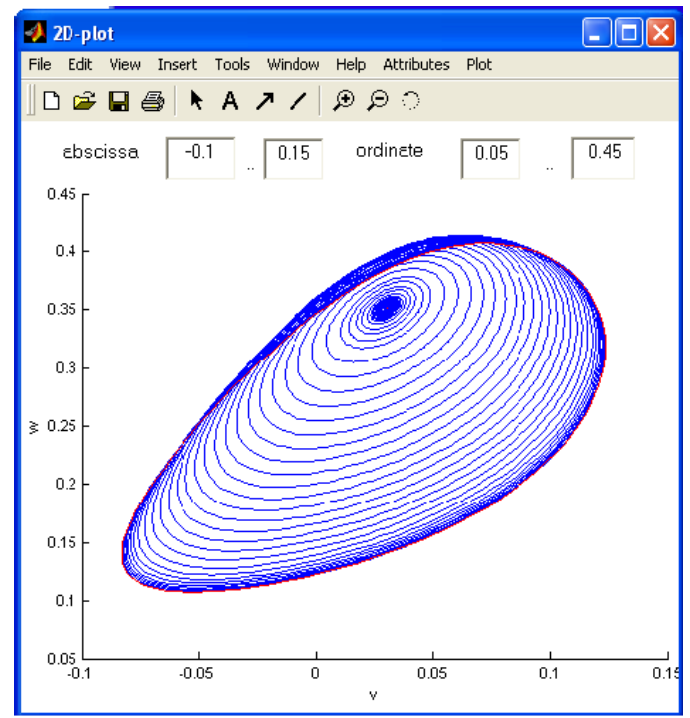

Fig. 3. LPC curve in phase space.

$z=0.1$ at $(0.04722,0.32564)$. We continue this equilibrium with free parameter $y$ for decreasing values of $y$. We find a Hopf $(\mathrm{H})$ bifurcation point at $y=0.075659$, two limit points (LP) at $y=-0.020727$ and $y=0.083257$ and a neutral saddle (H) at $y=0.033207$, see Fig. 2. There are stable equilibria before the first $\mathrm{H}$ point and after the second LP point and unstable equilibria between the first $\mathrm{H}$ point and the second LP point. The Lyapunov coefficient in the first Hopf point $l_{1}=16.47803$ is positive which means that the periodic orbits are born unstable. This Hopf point is used to start a limit cycle continuation. We choose $N=30$ test intervals and $m=4$ collocation points for the discretization. We detect a limit point of cycles LPC at $y=0.084569$. At this moment the stability is gained. Afterwards the stability is preserved but the period tends to infinity and the periodic orbits end in a homoclinic orbit. We select the LPC on this branch of limitcycles and free both $y$ and $z$ to continue the LPC curve through this LPC point. This curve is seen in Fig. 2 as the curve on the right of the LPC point and is also plotted in Fig. 3 in phase space. We note that it shrinks to a single point.

\section{References}

1. Allgower, E.L.,Georg, K.: Numerical Continuation Methods: An introduction, Springer-Verlag (1990)

2. Beyn, W.J., Champneys, A., Doedel, E., Govaerts, W., Kuznetsov, Yu.A., Sandstede, B.: Numerical continuation and computation of normal forms. In: B. Fiedler, G. Iooss, and N. Kopell (eds.) "Handbook of Dynamical Systems : Vol 2, Elsevier (2002) 149-219. 
3. Bykov, V.I., Yablonski, G.S., Kim V.F.: On the simple model of kinetic selfoscillations in catalytic reaction of $\mathrm{CO}$ oxidation, Dokl. Akad. Nauk SSSR 242(3)(1978) 637-639

4. De Boor,C. and Swartz, B.: Collocation at Gaussian points, SIAM Journal on Numerical Analysis 10(1973) 582-606.

5. Dhooge, A., Govaerts, W., Kuznetsov Yu.A.: Matcont: A matlab package for numerical bifurcation analysis of ODEs, to appear in TOMS (2003).

6. Doedel, E.J., Champneys, A.R., Fairgrieve, T.F., Kuznetsov, Yu.A., Sandstede, B., Wang, X.J., Auto97-Auto2000 : Continuation and Bifurcation Software for Ordinary Differential Equations (with HomCont), User's Guide, Concordia University, Montreal, Canada (1997-2000). (http://indy.cs.concordia.ca).

7. Doedel, E.J., Govaerts W., Kuznetsov, Yu.A.: Computation of Periodic Solution Bifurcations in ODEs using Bordered Systems, to appear in SIAM Journal on Numerical Analysis (2001).

8. Govaerts, W.: Numerical Methods for Bifurcations of Dynamical Equilibria, SIAM, Philadelphia (2000).

9. Khibnik, A.I., Bykov, V.I., Yablonski, G.S.: 23 phase portraits of the simplest catalytic oscillator, J. Fiz. Khim. 61(1987) 1388-1390

10. Kuznetsov, Yu.A.: Elements of Applied Bifurcation Theory, 2nd edition, SpringerVerlag, New York (1998)

11. Kuznetsov, Yu.A., Levitin, V.V., CONTENT: Integrated Environment for analysis of dynamical systems. CWI, Amsterdam (1997): ftp://ftp.cwi.nl/pub/CONTENT

12. MATLAB, The Mathworks Inc., http://www.mathworks.com

13. Mestrom, W.: Continuation of limit cycles in matlab, Master Thesis, Mathematical Institute, Utrecht University, The Netherlands (2002).

14. Morris, C., Lecar,H.: Voltage oscillations in the barnacle giant muscle fiber,Biophys J. 35 (1981) 193-213

15. Riet, A.: A Continuation Toolbox in matlab, Master Thesis, Mathematical Institute, Utrecht University, The Netherlands (2000).

16. Terman, D.: Chaotic spikes arising from a model of bursting in excitable membranes, Siam J. Appl. Math. 51 (1991) 1418-1450.

17. Terman, D.: The transition from bursting to continuous spiking in excitable membrane models, J. Nonlinear Sci. 2, (1992) 135-182. 